DOI: 10.1002/ ((please add manuscript number))

Article type: Full Paper

\title{
Effects of Delocalized Charge Carriers in Organic Solar Cells: Predicting Nanoscale Device Performance from Morphology
}

Adam G. Gagorik, Jacob W. Mohin, Tomasz Kowalewski, Geoffrey R. Hutchison*

Dr. A. G. Gagorik, Prof. G. R. Hutchison

University of Pittsburgh, 219 Parkman Avenue, Pittsburgh, PA 15260

E-mail: geoffh@pitt.edu

J. W. Mohin, Prof. T. Kowalewski

Carnegie Mellon University, 4400 Fifth Avenue, Pittsburgh, PA 15213

E-mail: tomek@andrew.cmu.edu

Keywords: charge transport, conducting polymers, organic electronics, solar cells

Monte Carlo simulations of charge transport in organic solar cells were performed for ideal and isotropic bulk heterojunction morphologies while altering the delocalization length of charge carriers. Previous device simulations have either treated carriers as point charges or with a highly delocalized mean-field treatment. Our new model of charge delocalization leads to weakening of Coulomb interactions and more realistic predicted current and fill factors at moderate delocalization, relative to point charges. We find that charge delocalization leads to significantly increased likelihood of escaping interface traps. In isotopic two-phase morphologies, increasing the domain sizes leads to slight decreases in predicted device efficiencies. We previously showed that tortuous pathways in systems with small domain sizes can decrease device performance in thin film systems. However, the diminishing effects of Coulomb interactions with delocalization and efficient separations of excitons by small domains make morphological effects less pronounced. We emphasize that delocalization, which has largely been ignored in past simulations, is an important parameter to consider and optimize when choosing materials for organic solar cells.

\section{Introduction}

A strong theoretical framework of charge transport in organic materials ${ }^{[1,2]}$ is needed to improve the efficiencies of organic field effect transistors (OFETs) $)^{[3,4,5,6,7]}$, organic light emitting diodes $(\mathrm{OLEDs})^{[8,9]}$, and organic photovoltaics $(\mathrm{OPVs})^{[10,11,12,13,14,15]}$. The promise is that these devices 
will be cheaper and more environmentally friendly than their inorganic counterparts. Costs are may be low because devices can be manufactured using existing solution processing techniques ${ }^{[16,17,18]}$ and synthetically tailored ${ }^{[19]}$ to specific applications. However, the understanding of charge transport in disordered organic semiconductors is far from complete. Mechanisms of charge transport in organic materials are very different from crystalline inorganic materials, where band transport occurs. While band transport can be observed in pure molecular crystals at low temperature, charge transport is typically described by localized carrier hopping at room temperature. ${ }^{[20]}$. The charge carriers are localized to molecular sites through disorder of the morphology and electron-phonon coupling. In contrast, highly delocalized carriers move through the energy bands of an inorganic material, yielding a high charge carrier mobility.

More efficient charge delocalization is therefore a major goal of molecular design in conjugated materials. Although the carriers are localized, some degree of delocalization can still play a critical role, especially in OPVs, where Coulomb bound interface traps easily form between "separated" carriers. For example, charge delocalization between $\pi$-stacked benzenes has been observed ${ }^{[21]}$.

Delocalization has also been suggested as a mechanism for efficient long-range charge separation in organic semiconductors ${ }^{[22,23]}$. For example, well-ordered, semi-crystalline domains likely lead to charge delocalization and improved device performance in OPVs. Single-junction devices with well ordered morphologies have been reported with very large fill factors (76-80\%) and power conversion efficiencies of up to $8.7 \%^{[24]}$. A complete picture of the role of crystallinity is not yet clear. While charge transport is enhanced by crystalline domains, exciton transport may be inhibited in well ordered systems ${ }^{[25]}$. This additional level of complexity is due to the dominance of exciton diffusion occurring by an intermolecular mechanism. In fact, exciton delocalization in regioregular P3HT is estimated to be as low as 1 to $2 \mathrm{~nm}$, and therefore may not play a major role in charge separation ${ }^{[26]}$. Studying the interplay of delocalization with electrostatic interactions between carriers may help elucidate these effects.

The combination of electrostatics and delocalization can play a major role in the charge separation process. For example, along with hot charge transfer (CT) states, calculations show that charge delocalization can lead to ultra fast ( $<100 \mathrm{fs}$ ) charge separation via the lowering of Coulomb bar- 
riers $^{[27]}$. While delocalization can be treated very accurately in quantum calculations ${ }^{[28,26,27]}$, the effect is generally ignored in meso-scale simulations of charge transport. In such simulations, highly accurate treament of electrostatic effects is achieved by largely ignoring the effects of delocalization and treating carriers as point charges ${ }^{[29,30,31,32]}$.

Since previous simulations have treated carriers exclusively as point charges or not at all, our primary goal was to add delocalization to Monte Carlo charge transport methods to more accurately represent carrier electrostatic interactions. To address these questions, we have incorporated delocalization into our existing Monte Carlo model ${ }^{[30,29,31,32]}$ of charge transport in OPVs. We have used the Monte Carlo model to examine a series of four isotropic two-phase morphologies with increasing domain size, three ideal morphologies based on mathematical minimal surfaces, and morphologies based on bands. We have not yet considered how delocalization effects transport for every possible mixed phase morphology. Such effects are likely worth further investigating.

In this work, the effects of delocalization have been examined by varying the delocalization length of the carriers, from 0.75 to $1.50 \mathrm{~nm}$, which are compared to simulated point charges and non-interacting charges. These length scales are comparable to those found from quantum calculations. ${ }^{[28,26,27]}$ As a result, transport behavior is affected on the length scale of the exciton dissociation radius ( 20-30 $\mathrm{nm})$. We have not yet addressed various questions such as how delocalization may be different in the donor and acceptor phases, anisotropy of delocalization, or how delocalization may vary during transport. For example, upon initial absorption of a photon, an exciton may be highly delocalized, and then localize after a very short time period (a few ps) due to electron-phonon coupling. Such topics are clearly relevant for future simulation efforts.

We have used our Monte Carlo simulations to predict current-voltage curves (IV Curves) of a solar cell device. From the IV curves we compute the efficiency of each system by calculating fill factors, a common figure of merit for solar cells. The fill factor $(F F)$ is the ratio of the maximum power that can be extracted from the cell to the theoretical power, as shown in Equation 1:

$$
F F=\frac{v_{m p} \times i_{m p}}{v_{o c} \times i_{s c}} \times 100 \%
$$

In Equation 1, $i_{m p}$ is the current at maximum power, $v_{m p}$ is the voltage at maximum power, $i_{s c}$ is the short circuit current, and $v_{o c}$ is the open circuit voltage. The fill factor ranges between 0 
and $100 \%$, and can be viewed as the ratio of the areas of two rectangles - a smaller one formed by $i_{m p}$ and $v_{m p}$, and a large one formed by $i_{s c}$ and $v_{o c}$. The closer the fill factor is to $100 \%$, the more square (ideal) the IV curve, and the more efficient the performance of the solar cell. In a real device, the solar cell is connected to an external load, which has a resistance, $R$. Only when $R$ is the ratio $v_{m p} / i_{m p}$ can the maximum power be extracted from the device. The short circuit current $i_{s c}$ corresponds to $R=0$, while the open circuit voltage corresponds to $R=\infty$. While the fill factor gives information on IV curve shape, solar cells with similar fill factors can have different $p_{m p}$. For example, a device that poorly passes current can have a small $i_{s c}$ and $i_{m p}$, yet appear to have a good fill factor. Therefore, it is important to consider multiple measures of performance, as discussed below.

\section{Computational Methods}

\subsection{Lattice}

The model consists of a lattice of cubic sites, with each site representing neutral molecules, 1.0 $\mathrm{nm}^{3}$ in size, approximately the same size as an isolated fullerene molecule. This is therefore the smallest possible lattice without treatment of atomic-scale details.

In this work, we have used a lattice of $192 \times 192 \times 32 \mathrm{~nm}^{3}$. Charge carriers, such as holes and electrons, can occupy the sites. Holes are to be viewed as an electron missing from the highest occupied molecular orbital (HOMO) of a molecule. Electrons are to be viewed as an excess electron in the lowest unoccupied molecular orbital (LUMO). When an electron and hole occupy the same site, they are an exciton. Excitons can recombine to form unoccupied sites.

\subsection{Exciton Injection}

The simulation begins by injecting excitons randomly in the system, as a model for photo-generated electronic excitations. We have assumed that the system is ideal in that all sites have the same probability to form an exciton, regardless of thickness. In the future, we will relax these assumptions, 
to allow for non-uniform thickness or self-adsorption in deeper layers. For now, we have used an injection probability estimated from the AM1.5 solar spectrum, and is proportional to the surface area of the device. For a $256 \times 256 \mathrm{~nm}^{2}$ area, we have estimated an injection probability of $10^{-3}$ $\mathrm{ps}^{-1}$. This injection probability is scaled by a factor of $192^{2} / 256^{2}=0.5625$ for an area of 192 $\times 192 \mathrm{~nm}^{2}$. While optical effects may change the intensity distribution (e.g., due to absorbance attenuating light in the film, and differences in refractive indices between the p-type and n-type phase) these are currently ignored.

\subsection{Recombination}

Recombination is also allowed to occur at a fixed probability for excitons in the system. Unlike injections, a fixed recombination probability does not directly correspond to a fixed rate because excitons are only considered when holes and electrons occupy the same site. A constant probability is a simplification; recombination in organic solar cells can happen by many mechanisms with different timescales ${ }^{[33]}$. For example, in a bimolecular recombination mechanism, the Langevin expression predicts recombination rate to depend upon the hole/electron mobilities, the electron/hole concentrations, and the intrinsic carrier concentration ${ }^{[33]}$. We have used a fixed recombination probability of $10^{-4} \mathrm{ps}^{-1}$, inspired by kinetic Monte Carlo simulations ${ }^{[34,35,36]}$. Recombination is performed at the end of each step. All electrons and holes occupying the same site are allowed to recombine at the fixed rate given above. The units on $10^{-4} \mathrm{ps}^{-1}$ mean that, if, on average, only one exciton is present at the end of every Monte Carlo step, then it would take $10 \mathrm{~ns}$ for an exciton to recombine. There are frequently more than one exciton at the end of each step. In practice, this fixed rate is not realized because the average number of excitons present in the simulation is highly dependent upon charge concentration. The concentration will change with voltage. This means charges are less likely to combine at high voltages (carriers are being pulled apart) and more likely to recombine at $v_{o c}$. At $v_{o c}$, the external bias balances the internal bias. With only Coulomb interactions present, pulling separated carriers apart becomes difficult; it is likely for excitons to reform and recombine. 


\subsection{Energy Evaluations}

Charges hop between adjacent sites using the Metropolis criterion. The probability to hop between sites is $H_{A B} e^{-\beta \Delta E}$ if $\Delta E \leq 0$. If $\Delta E>0$, the probability to hop between sites is $H_{A B} . H_{A B}$ can be thought of as a coupling constant representing the approximate electronic overlap between molecular wave functions. However, more practically, $H_{A B}$ enforces carriers to remain stationary a fraction of the time when energy changes are negative. We have used $H_{A B}=1 / 3$, independent of hopping direction. The value used determines the overall magnitude of the current calculated, and approximates experimental current in OFETs ${ }^{[32]}$. Directional independence is an approximation that may not be representative of many systems, where charge transfer rates may be anisotropic (i.e., along $\pi-\pi$ stacks). For example, charge transfer efficiency will be preferred along stacked domains, although in an amorphous device, such directional effects would be expected to average out. Additionally, coupling would be different among differing materials. $H_{A B}$ is made exponentially smaller, $H_{A B}=1 / 27$, for a two-site hop. This is made to simulate an exponentially less likely tunneling event in variable-range hopping. Carriers are considered individually for trial moves. Sites are assigned energies representing the HOMO and LUMO energies of the molecules. There are three main contributions: the donor/acceptor offset, the overall device potential between electrodes (including differences between metal work functions), and the Coulomb interaction between charges.

The sites are classified as donor or acceptor, according to the system morphology chosen, as discussed below. The donor/acceptor LUMO and HOMO are offset by a constant energy $\Delta E_{L L}=$ $\Delta E_{H H}=0.5 \mathrm{eV}$. This value is slightly larger than the Coulomb interaction between two opposite charges spaced $1 \mathrm{~nm}$ apart $0.411 \mathrm{eV}$. It provides a driving force to separate carriers at the interface. and the open-circuit voltage $V_{O C}$ is idealized at an optimum of $\sim 1.5 \mathrm{~V}$, to consider the possible efficiencies of ideal materials. Realistic experimental $V_{O C}$ values are usually 0.5-0.8 V. Next, a linear electrostatic potential is applied between the electrodes, using the potential difference $\Delta V=V_{f}-V_{i}$ and width of the device, $L$. This potential includes the external and intrinsic bias. To construct an IV curve, this potential is varied from -2.0 to $2.0 \mathrm{~V}$ to pass the $V_{O C}$. 

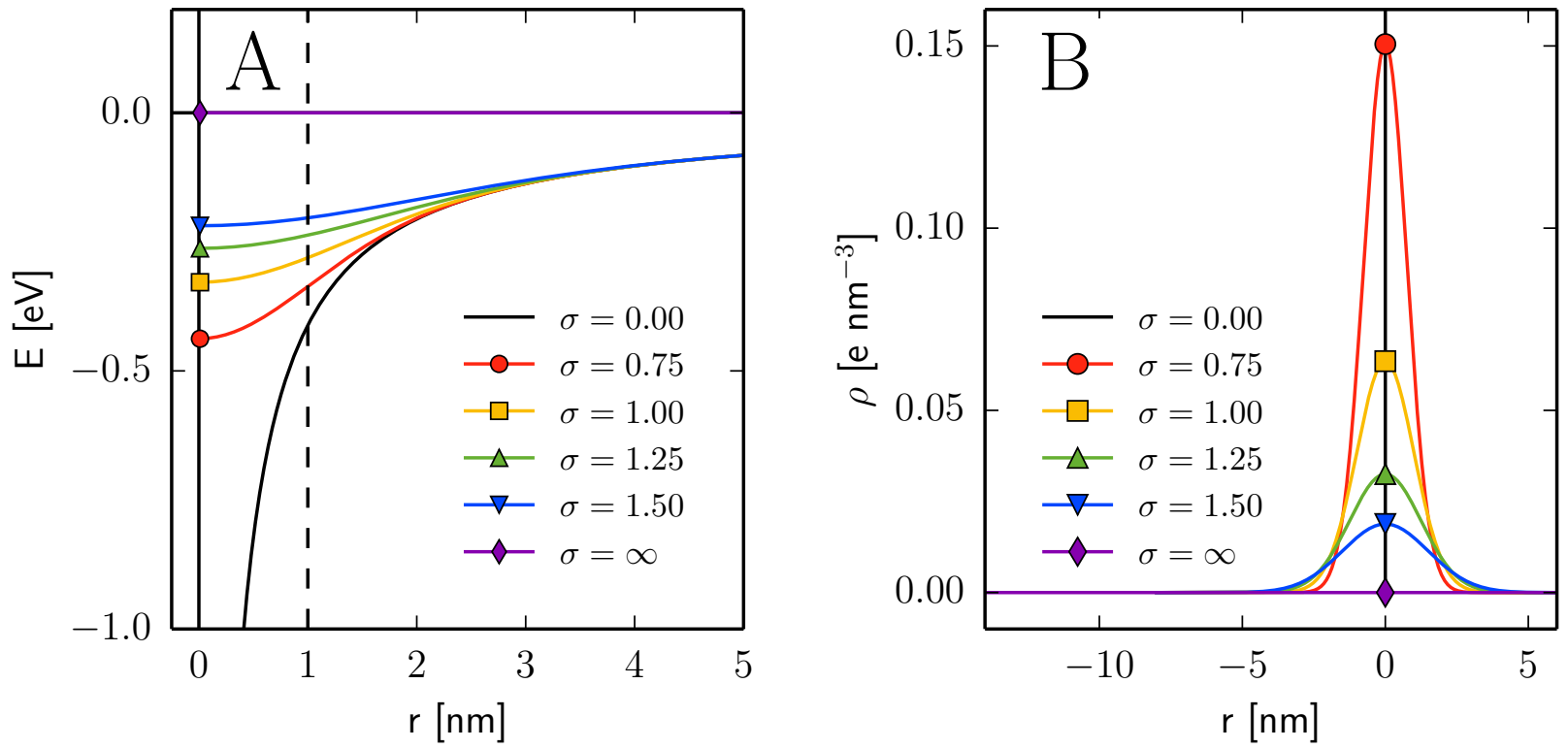

Figure 1: (A) Coulomb potential of a delocalized negative charge located at the origin as a function of r. (B) Charge density of the delocalized charge. As the delocalization parameter, $\sigma$, increases, the Coulomb interaction becomes weaker. Point charges correspond to a delocalization parameter of $\sigma=0 \mathrm{~nm}$. Non-interacting charges are represented by $\sigma=\infty$.

\subsection{Electrostatic Interactions}

Disorder is added to the system using the Coulomb interaction, as shown in Equation 2. In Equation 2, $N$ is the number of charges (carriers or charged defects), $q$ is the charge, $r_{i j}$ is the distance between charges $i$ and $j$, and $\epsilon=3.5$ is the dielectric constant. For every Monte Carlo move, the Coulomb interaction must be calculated for the initial and final state - around $2 \times N \times N_{\text {steps }}$ times. Even with a cutoff of $50 \mathrm{~nm}$ and lookup table for inter-cell distances, the brute force evaluation of Equation 2 is the most time consuming step of the simulation, because it scales as $N^{2}$. Therefore, a parallel GPU implementation of the Coulomb sum was used to make the calculation feasible. With the GPU code, carrier numbers of 10,000 or more can be handled.

$$
V_{C}\left(\mathbf{r}_{j}\right)=\sum_{i=0}^{N} \frac{q}{4 \pi \epsilon \epsilon_{0}\left|\mathbf{r}_{i j}\right|}
$$

Equation 2 describes the interaction between point charges. To compute the potential due to a charge distribution, one uses Poisson's Equation, $\nabla^{2} V=\rho /\left(\epsilon \epsilon_{0}\right)$, where $\rho$ is the charge density. 
When using a charge density described by spherically symmetric Gaussians (Equation 3), an analytical solution can be found (Equation 4). The result is is simply the Coulomb law multiplied by the error function, with a parameter $\sigma$ describing the delocalization length.

$$
\begin{gathered}
\rho\left(\mathbf{r}_{j}\right)=\sum_{i=0}^{N} \frac{q}{\sigma^{3}(2 \pi)^{3 / 2}} e^{-\frac{\left|\mathbf{r}_{i j}\right|^{2}}{2 \sigma^{2}}} \\
V_{C}^{\operatorname{erf}}\left(\left|\mathbf{r}_{j}\right|\right)=\sum_{i=0}^{N} \frac{q}{4 \pi \epsilon \epsilon_{0}\left|\mathbf{r}_{i j}\right|} \operatorname{erf}\left(\frac{\left|\mathbf{r}_{i j}\right|}{\sigma \sqrt{2}}\right)
\end{gathered}
$$

The error function only modifies the Coulomb interaction at short range. For example, when $\sigma=$ $1 \mathrm{~nm}, 0.95 \leq V_{C}^{\mathrm{erf}} / V_{C} \leq 1$ at $r \geq 2 \mathrm{~nm}$. However, at short distances, the Coulomb potential is significantly diminished. For example, when $\sigma=1 \mathrm{~nm}, 0.0 \leq V_{C}^{\mathrm{erf}} / V_{C} \leq 0.68$ at $r \leq 1 \mathrm{~nm}$. This effect can be seen in Figure 1A, where the potential at each value of $\sigma$ converges to the Coulomb potential at large $r$ (i.e., $\geq 3 \mathrm{~nm}$ ). The charge density as a function of $r$ is shown in Figure $1 \mathrm{~B}$. The charge density was chosen such that each Gaussian integrates to a unit charge. Note that this solution to Poisson's equation can only be obtained in 3D, so could not be applied to our previous simulations $^{[30]}$ on 2D monolayer OPVs. The use of spherically symmetric charge distributions is an oversimplification. In reality, molecular orbitals with angular momentum are not spheres. Anisotropy will be added in the future, for example, by considering Gaussians ellipsoids.

The choice of the $\sigma$ delocalization lengths of $0.75 \mathrm{~nm}$ to $1.5 \mathrm{~nm}$, as discussed above, corresponds to a range found through quantum mechanical calcualtions. ${ }^{[28,26,27]}$ Moreover, as seen in Figure 1A, with the $1.0 \mathrm{~nm}$ grid spacing between molecules, $\sigma$ ranging from $0.75 \mathrm{~nm}, 1.0 \mathrm{~nm}$, and $1.25 \mathrm{~nm}$, roughly span the energetic range between point charges and $\sigma=1.5 \mathrm{~nm}$. Smaller $\sigma$ values experience almost the same electrostatic interactions at this grid spacing as point charges, and vanishingly small probabilities of acceptable Monte Carlo moves, as indicated in Table S1 and illustrated in Figure S5. Since the spatial resolution of our Monte Carlo grid roughly corresponds to a molecular size, while decreased delocalization lengths may be interesting, such considerations would require treatment of atomic-level (i.e., quantum) details. 
A) gyroid

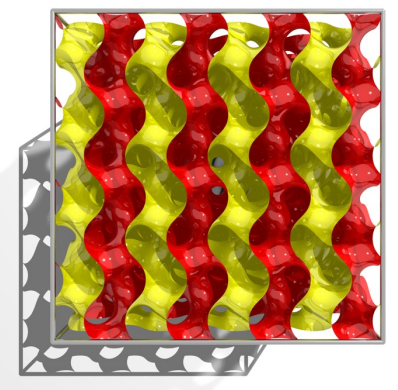

E) iso3

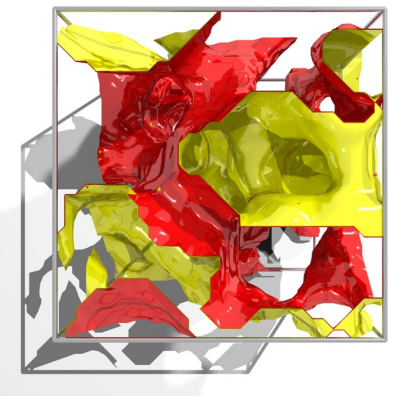

B) p-surface

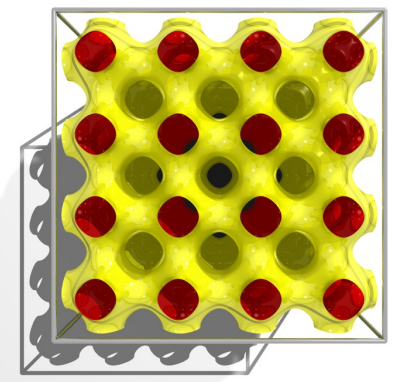

F) iso4

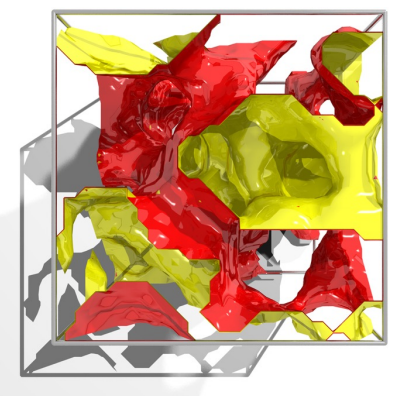

C) d-surface

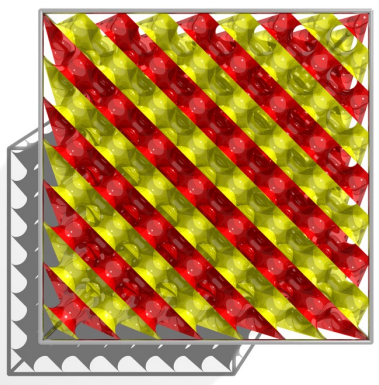

G) iso6

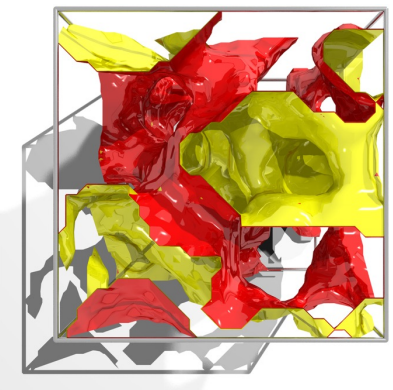

D) band4

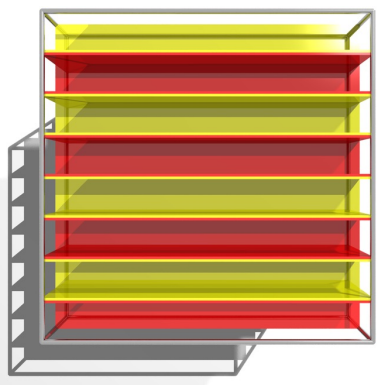

H) iso9

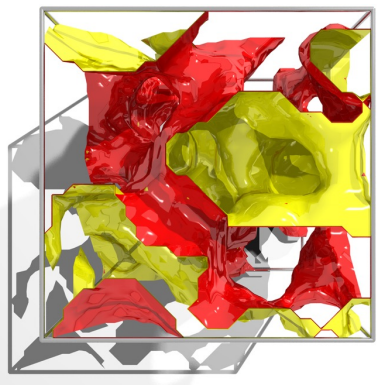

Figure 2: $(32 \mathrm{~nm})^{3}$ slices of all morphologies studied. Full systems were $192 \times 192 \times 32 \mathrm{~nm}^{3}$ and shown in the supporting information.

\section{Systems}

Morphologies were constructed with a domain of $192 \times 192 \times 32 \mathrm{~nm}^{3}$, or 1,179,648 sites. Periodic boundary conditions were not used, to properly include finite size effects in real materials, such as surface trapping. All morphologies consisted of a donor and an acceptor phase whose frontier molecular orbital energies were offset arbitrarily by $0.5 \mathrm{eV}$, as described above. Device performance can be affected by many parameters, such as mobility and connectivity, and is not limited to just charge delocalization. Therefore, we have considered ideally connected morphologies and morphologies more representative of bulk heterojunctions. Realistic, isotropic morphologies were simulated, and compared with "ideal" morphologies constructed from equations of triply periodic minimal surfaces ${ }^{[37]}$, shown in Figure 2. Such surfaces are free of self intersections and contain perfect connectivity to the electrodes. The minimal surfaces chosen, which are of the form $f(x, y, z)=0$, were the gyroid (Eq. 5, Figure 2A), p-surface (Eq. 6, Figure 2B), and d-surface 
(Eq. 7, Figure 2C).

$$
\begin{gathered}
\cos \left(k_{x} x\right) \sin \left(k_{y} y\right)+\cos \left(k_{y} y\right) \sin \left(k_{z} z\right)+\cos \left(k_{z} z\right) \sin \left(k_{x} x\right)=0 \\
\cos \left(k_{x} x\right)+\cos \left(k_{y} y\right)+\cos \left(k_{z} z\right)=0 \\
\sin \left(k_{x} x\right) \sin \left(k_{y} y\right) \sin \left(k_{z} z\right)+ \\
\sin \left(k_{x} x\right) \cos \left(k_{y} y\right) \cos \left(k_{z} z\right)+ \\
\cos \left(k_{x} x\right) \sin \left(k_{y} y\right) \cos \left(k_{z} z\right)+ \\
\cos \left(k_{x} x\right) \cos \left(k_{y} y\right) \sin \left(k_{z} z\right)=0
\end{gathered}
$$

In Equations 5, 6, and 7, $k$ is an angular wavenumber, defining the periodicity of the surface. We choose a $k$ that corresponds to a wavelength $\lambda=8 \mathrm{~nm}$, as shown in Equation 8 , where $L$ is a dimension of the grid, and $n$ is the number of periods along the dimension. Only systems where ( $k=k_{x}=k_{y}=k_{z}$ ) were studied, and the minimum possible distance between surface boundaries is $\frac{\lambda}{2}=4 \mathrm{~nm}$.

$$
\begin{gathered}
\lambda=\frac{L}{n}=\frac{192}{8}=\frac{32}{4}=8 \\
k=\frac{2 \pi}{\lambda}=\frac{2 \pi}{8}=\frac{\pi}{4}
\end{gathered}
$$

In practice, sites were assigned by evaluating $f(x, y, z)$ on the domain and thresholding. Donor sites were placed where $f(x, y, z)>=0$, while acceptor sites were placed where $f(x, y, z)<0$. This assignment makes the zero-level contour-isosurface of $f(x, y, z)$ the boundary between donor and acceptor sites.

The last ideal morphology studied was the "band" morphology. The band morphology is a checkerboard pattern of alternating donor and acceptor sites in the yz-plane, extended along xdirection. Two band morphologies were used. The band4 morphology has a checker size of $4 \times 4$ $\mathrm{nm}^{2}$. The band8 morphology has a checker size of $8 \times 8 \mathrm{~nm}^{2}$. Figure $2 \mathrm{D}$ shows a $(32)^{3} \mathrm{~nm}^{3}$ slice of the band 4 morphology.

Traditional isotropic morphologies, intended to simulate a random organic bulk heterojunction, are also shown in Figure 2. Such isotropic morphologies were constructed using an algorithm 
discussed previously ${ }^{[30]}$, extended into three dimensions. In the algorithm, a grid of random noise is constructed in the domain and then convoluted with a three-dimensional Gaussian of desired width, $\sigma$. These real valued sets are then converted into binary maps by a thresholding rule, with zeros corresponding to D (donor) and ones corresponding to A (acceptor) phases. The average domain size in such maps is set by the value of $\sigma$. To produce morphologies with equal volume fractions of both phases $\left(\phi_{1}=\phi_{2}=50 \%\right)$, the thresholding rule was applied at the $50 \%$ level of the cumulated probability of the normalized distribution of intensities. In these morphologies, the domain size $\left\langle L_{p}\right\rangle$ is approximately twice the size of $\sigma$, such that the $\sigma=3,4,6,9 \mathrm{~nm}$ leads to domain sizes of $6,8,12,18 \mathrm{~nm}$. Four isotropic systems were studied, named iso3, iso4, iso6, and iso9. An important metric of the morphologies is the inter-facial area between phases, which is correspondingly $196.04,123.31,104.39,71.52 \mathrm{~m}^{2} \mathrm{~cm}^{-3}$. The iso3 morphology domain size is comparable to the minimum curvature morphologies' minimum distance between phases of 4 nm. This excludes the band8 morphology, which has a domain size more comparable to the iso4 system.

Although the isotropic two-phase morphologies represent a mixed phase system already, we probed the effect of adding inter-dispersed acceptor sites in the donor material of the iso9 system. Such "mixed phases" occur when individual fullerene molecules disperse into the polymer donors. In this case, called iso9p, 1179 sites $(0.2 \%)$ of the donor phase was changed to acceptor material at randomly chosen locations. In the end, however, one must keep in mind that a real macroscopic device will be a combination of multiple regions with somewhat different morphologies, and thus should view our simulation as a subset of such a device.

While our model could consider more phases, that is not our current focus. In the future, a few avenues will be pursued to better differentiate phases. For example, multiple energetic offsets will be used to create a 3 or more domain system. Anisotropic transfer couplings $\left(H_{A B}\right.$, discussed above) will be used to consider multi-scale size effects, such as crystalline regions with larger transfer rates. While we use a rectangular lattice, others will be implemented, to better probe the effects of crystallization. Though this would modify transfer probability through changing the energetic calculation, the more direct approach would be changing $H_{A B}$. Finally, we will consider 

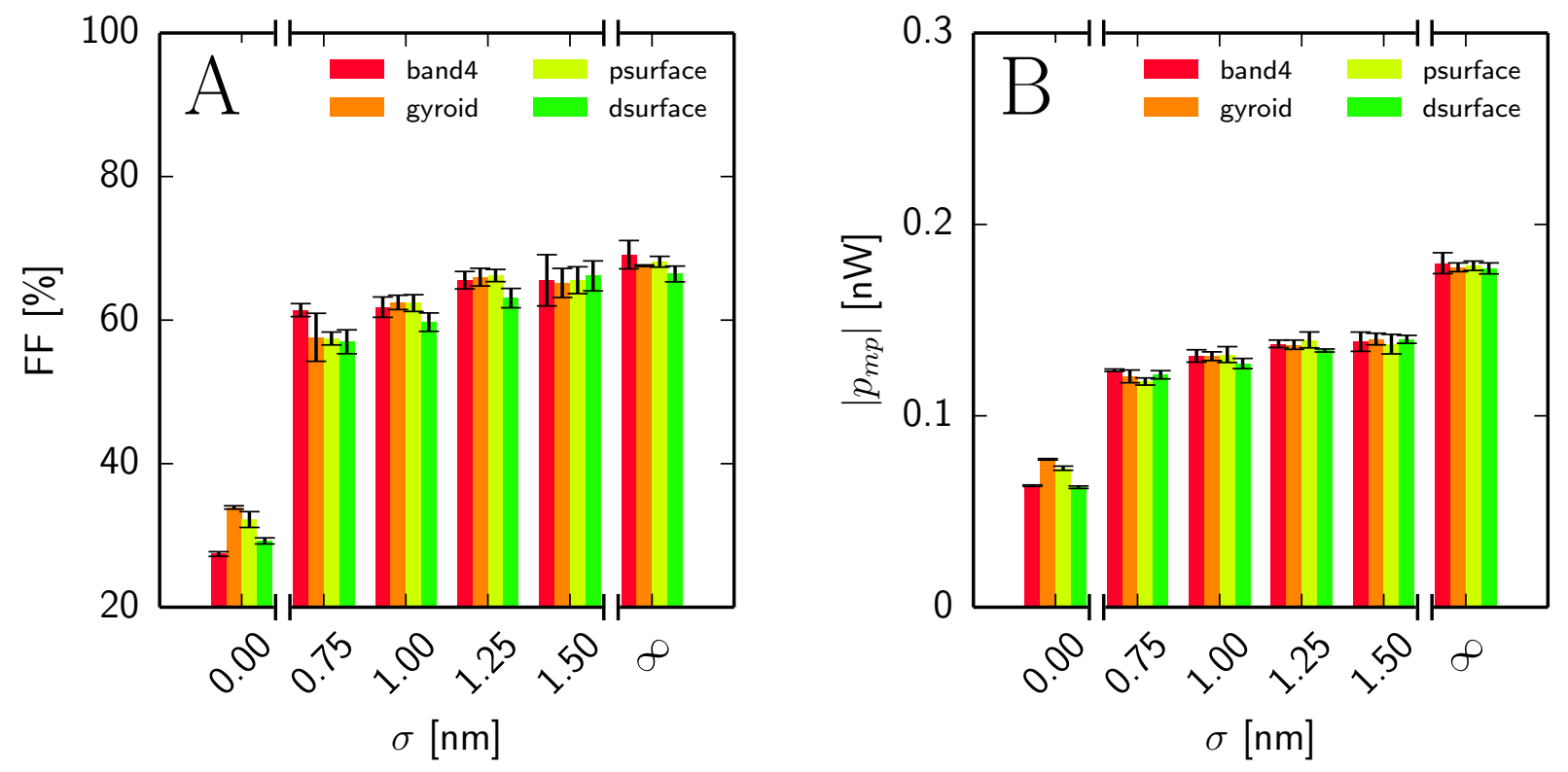

Figure 3: (A) Calculated fill factor as a function of delocalization length $(\sigma)$ for ideal morphologies. (B) Calculated maximum power as a function of delocalization length $(\sigma)$ for ideal morphologies.

a multi-region cell, where different regions contain differing order parameters, such as domain size.

\section{Results and Discussion}

Figure 3A shows the computed fill factors for ideal morphologies. Aside from the band8 morphology, each ideal system has the same domain size of $4 \mathrm{~nm}$. As the delocalization is increased from point charges $(\sigma=0.00 \mathrm{~nm})$ to delocalized charges $(\sigma=0.75 \mathrm{~nm})$, the average $F F$ increases from $35 \%$ to $58 \%$. The FF continues to increase, less dramatically, as the delocalization is increased to $\sigma=1.50 \mathrm{~nm}$. Non-interacting charges, which can be viewed as infinitely delocalized carriers in this model, yield devices with the best $F F(70 \%)$. While there is no significant trend in morphology with delocalized charges, the trend with point charges is gyroid $<\mathbf{d}$-surface $<$ p-surface $<$ band4. Small differences exist, but are not statistically significant with delocalized charges. Figure 3B shows the maximum power $\left(p_{m p}\right)$ for ideal morphologies. The $p_{m p}$ indicates the maximum work per second that can be performed by the solar cell. The maximum power and 

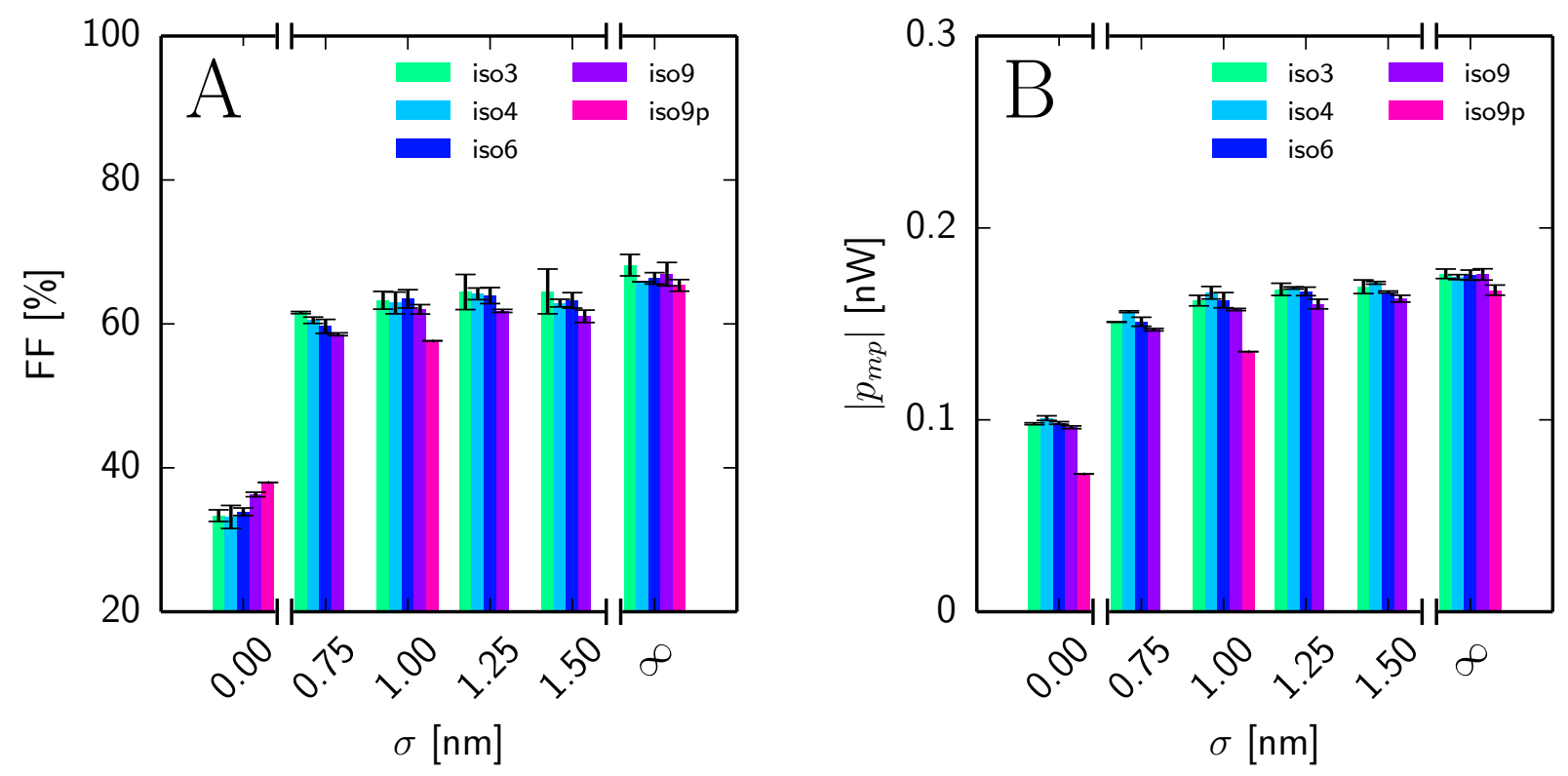

Figure 4: (A) Calculated fill factor as a function of delocalization length $(\sigma)$ for isotropic twophase morphologies. (B) Calculated maximum power as a function of delocalization length $(\sigma)$ for isotropic two-phase morphologies.

power conversion efficiency are both proportional to the fill factor, and thus increase with delocalization. The largest increases are seen when moving from point charges to delocalized charges, and from delocalized charges to non-interacting charges. With point charges, transport is hindered by energetic disorder, but as the energetic disorder is smoothed out by delocalization, the maximum power increases because more carriers can navigate to the electrodes. Interestingly, the predicted $i_{s c}$ is consistent across all ideal morphologies and delocalization lengths, but the $i_{m p}$ drives the increase in maximum power. This suggests that delocalization effects change the shape of the $I V$ curve, not simply increasing the overall current due to an increased number of carriers escaping interfacial pinning.

Figure 4A shows computed fill factors for isotropic two phase morphologies as a function of sigma. The iso4 and iso6 systems have very similar average domain size $(8.1,9.57 \mathrm{~nm})$. Within error, their $F F$ are very similar. As the delocalization of carriers increases, the device performance increases, much like the ideal morphologies. The most dramatic increase occurs between point charges and $\sigma=0.75 \mathrm{~nm}$, where the fill factors increase from $<40 \%$ to $>50 \%$. Since charge 
carriers in organic solar cells are not point charges, but delocalized, the former is unrealistic for such idealized morphologies, and the latter is more likely considering champion solar cells already achieve $F F$ approaching 70\%. However, the differences of $F F$ for $\sigma=0.75-1.50 \mathrm{~nm}$ is much less pronounced. Only when non-interacting charges $(\sigma=\infty)$ are used, does the $F F$ increase significantly. The fill factor decreases in iso9p relative to iso9 for $\sigma=1.00 \mathrm{~nm}$ and non-interacting charges. However, for point charges, the fill factor is not smaller. Figure $4 \mathrm{~B}$ shows $p_{m p}$ for isotropic morphologies, which increases with delocalization. The $p_{m p}$ is consistently smaller in iso9p relative to iso9. In the iso9p system, acceptor sites, termed "pepper," were randomly added in the donor phase, representing PCBM inter-dispersed in the P3HT phase. This is due a smaller $i_{m p}$ when pepper defects are present ( $v_{m p}$ does not vary between iso9 and iso9p). The pepper defects acts as electron traps, reducing the number of carriers that can escape to the electrodes.

When we compare the idealized mathematical minimal surfaces, such as gyroid, p-surface, and d-surface to the two-phase isotropic morphologies, such as iso3, iso4, etc., we note that the $i_{s c}$ and $i_{m p}$ are slightly lower. That is, the typical curved two-phase isotropic systems show greater predicted maximum power and thus power conversion efficiency than idealized systems. We suspect the increased interfacial surface area in these bulk-heterojunction morphologies, combined with efficient exciton separation and escape from the Coulomb-pinned interfaces with delocalized charges, lead to such high maximum powers. These results suggest that in materials with high delocalization and efficient exciton separation, traditional bulk-heterojunction morphologies may be at least as efficient as band or "comb" idealized morphologies due to high surface area.

In a previous study, on simulations of $2 \mathrm{D}$ monolayer devices, we found that decreasing the domain size leads to poorer charge extraction in thin films ${ }^{[30]}$. We argued that while smaller domain sizes lead to efficient exciton separation, small tortuous pathways can hinder charge extraction. The close proximity of charges and tortuous morphology to the electrodes lead to a disordered energy landscape with a limited number of viable pathways to the electrodes. The trend appears to reverse in larger bulk 3D systems. That is, smaller domains show improved device performance at each value of the delocalization parameter $\sigma$. However, we note that the effects are relatively small and within statistical errors. More replicas need to be simulated and averaged to elucidate the effect. 


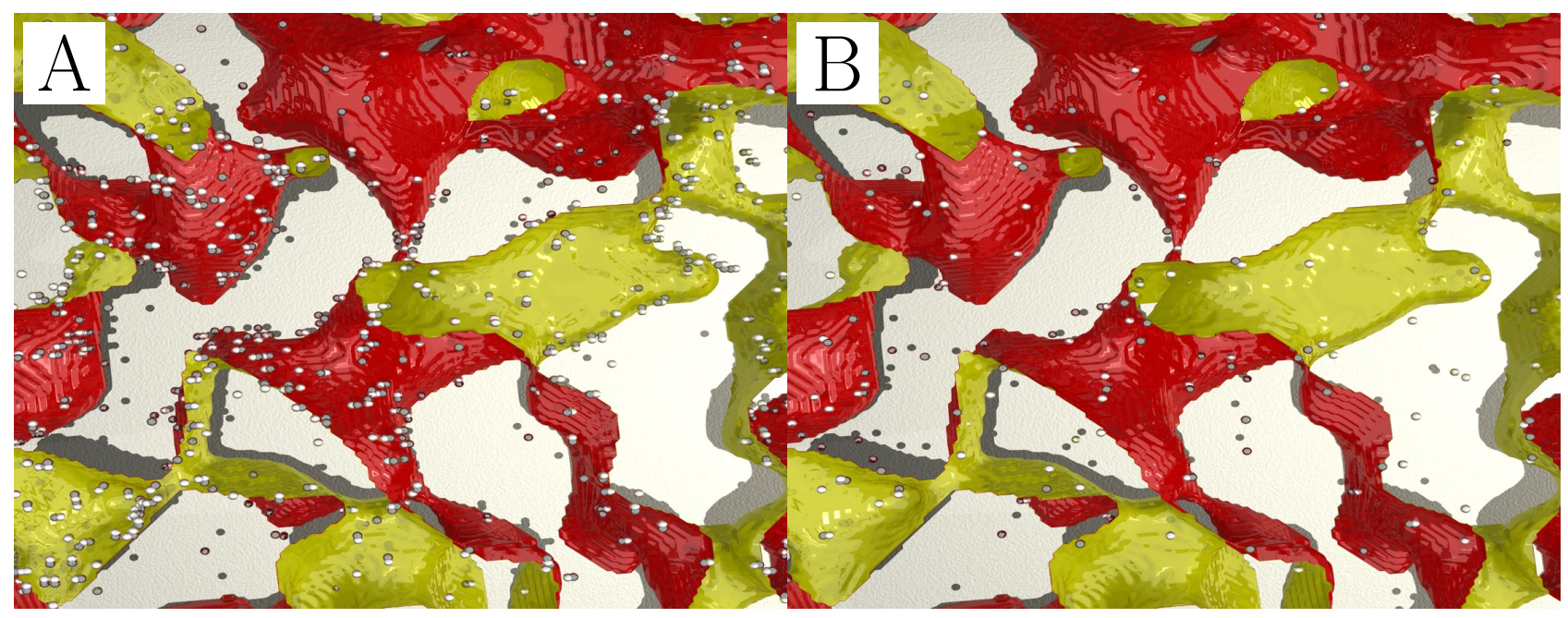

Figure 5: Snapshot of iso3 morphology at $v_{o c}$. Interface traps form more easily with less delocalization. Yellow and red represent donor and acceptor sites. Electrons and holes are shown as black and white spheres. (A) $\sigma=0.75 \mathrm{~nm}$ (B) $\sigma=1.50 \mathrm{~nm}$

Given the interplay between tortuous pathways and efficient exciton separation, the separation appears to win. That is, we propose that more pathways have opened up in the $3 \mathrm{D}$ environment than were present in our previous 2D thin film simulations, due to the increased dimensionality. Moreover, the decreased electrostatic interactions due to the delocalized electrostatic model likely increases the influence of efficient exciton separation.

Figure 5 shows snapshots of the Monte Carlo simulation for the iso3 morphology at $v_{o c}$. At $v_{o c}$, the potential between the electrodes balances the internal intrinsic bias. Therefore, there is very little incentive for excitons to separate and free carriers to travel to the electrodes. Recombination is the most likely fate of the carriers in this regime. However, the effects of charge delocalization are easily visualized at $v_{o c}$. When $\sigma=0.75 \mathrm{~nm}$ (Figure 5A), holes and electrons remain bound in interface traps. However, as the delocalization is increased to $\sigma=1.50 \mathrm{~nm}$ (Figure 5B), the number of bound interface traps decreases significantly. The energetics of escaping the interface trap are more favorable when the carriers are delocalized.

Consider, for example, an electron-hole pair trapped at an interface. The exciton has separated, putting the electron in the LUMO of the acceptor, and the hole in the HOMO of the donor. The Coulomb attraction between the hole and electron in dielectric continuum $(\epsilon \approx 3.5)$ separated by 
$1 \mathrm{~nm}$ is $0.411 \mathrm{eV}$, nearly 15.9 times larger than $\mathrm{kT}$ at $300 \mathrm{~K}(0.0259 \mathrm{eV})$. A potential difference of $1.0 \mathrm{~V}$ between electrodes separated by $256 \mathrm{~nm}$ only releases $0.00390 \mathrm{eV}$ of energy during the 1.0 $\mathrm{nm}$ hop out of this state. Given that the Coulomb attraction is only halved at $2.0 \mathrm{~nm}$, this means the hole and electron will "separate" only a minuscule fraction of the time $\left(e^{-\beta \Delta E}=0.0407 \%\right)$. Even if the carriers reach this state, it is extremely likely the charges fall back into the charge trap state, and possibly recombine. There must be other mechanisms to explain the efficient charge separation in organic materials.

The delocalization of charges can weaken the Coulomb interaction between trapped carriers. While a fully quantum treatment would be ideal, the interaction of spherically symmetric Gaussian charge distributions can be modeled using the standard Coulomb law and the error function (Eq. 4). Using this model, the probability to separate carriers, of only modest delocalization lengths ( $\sigma$ $=0.5-1.5 \mathrm{~nm}$ ) can increase dramatically. For example, a delocalization length of $1.0 \mathrm{~nm}$ dampens the Coulomb interaction by $\operatorname{erf}(1.0 / \sqrt{2})=68 \%$ at an electron hole separation of $1.0 \mathrm{~nm}$. The probability to escape the charge trap state increases by over a factor of $100(\Delta E=0.0806 \mathrm{eV}, \mathrm{P}=$ $4.42 \%$ ). These calculations are compiled in Table S1.

\section{Conclusion}

In summary, our simulations show decreased effect of morphological details and that charge delocalization leads to significantly increased charge extraction in OPVs. We propose that the weakening of energetic barriers, particularly in Coulomb interface traps leads to the efficient separation of charges. Multiple length scales are involved in assessing device performance, such as micronscale morphological effects and the nanoscale hole-electron radius of interaction. Delocalization increases the interaction radius, allowing charges to escape the electrostatic pull at the interface. This occurs well under the length scale of the morphology. The escaped charge carriers can more readily exit the system, increasing device parameters such as current, maximum power, and fill factor.

The predicted fill factors in 3D are overall larger (average FF $58 \pm 12 \%$ ) and closer to exper- 
imental champion devices ( $\mathrm{FF} \sim 60-70+\%$ ) than previous $2 \mathrm{D}$ thin film simulations ${ }^{[30]}$, where the predicted FF were unrealistically low for idealized devices. The predicted fill factors also depend less on the morphology, suggesting new materials with highly delocalized carriers (i.e., along the polymer chain, between chains, and between acceptor molecules) may be less sensitive to morphological differences between devices.

In thin films, the lattice easily becomes frustrated and the Coulomb interactions dominate the energy landscape. In 3D systems, more pathways open up, alleviating some of the frustration, and increasing the device efficiency. While the differences in morphology (domain size for isotropic systems and geometrical structure for ideal systems) did not play an important role in device efficiency, we note that a larger sample of morphologies, particularly those with mixed phases, must be examined in the future with more factors to differentiate them.

Considering charges in $\pi$-conjugated organic materials are known to be delocalized, this new delocalized electrostatic model is a much more realistic and computationally-efficient method to treat carrier-carrier interactions in organic solar cells and similar devices. Point charge models dramatically overestimate the interfacial charge pinning, and mean-field methods significantly underestimate such effects.

Seeking materials that can effectively allow charges to delocalize over multiple molecules, especially near an interface, is a promising route to create efficient solar cells. While a material with an overall higher dielectric constant will allow for a more efficient device (i.e., inorganic materials), it may be that the weakening of Coulomb interactions near an interface is the dominant player in organic materials and should be the focus of future materials design.

\section{Supporting Information}

Supporting Information is available from the Wiley Online Library or from the author.

\section{Acknowledgements}

We thank the University of Pittsburgh, Carnegie Mellon University, and Bayer Materials Science for financial support. This research was supported in part by the University of Pittsburgh Center for Simulation and Modeling through the supercomputing resources provided.

Received: ((will be filled in by the editorial staff))

Revised: ((will be filled in by the editorial staff)) Published online: ((will be filled in by the editorial staff)) 


\section{References}

[1] H. E. Katz, J. Huang, Ann. Rev. Mater. Res. 2009, 39, 71-92.

[2] T. B. Singh, N. S. Sariciftci, Ann. Rev. Mater. Res. 2006, 36, 199-230.

[3] J. Smith, W. Zhang, R. Sougrat, K. Zhao, R. Li, D. Cha, A. Amassian, M. Heeney, I. McCulloch, T. D. Anthopoulos, Adv. Mater. 2012, 24, 2441-2446.

[4] S. Kola, J. Sinha, H. E. Katz, J. Polym. Sci. Part B: Polym. Phys. 2012, 50, 1090-1120.

[5] H. Sirringhaus, Proc. IEEE 2009, 97, 1570-1579.

[6] M. Mas-Torrent, C. Rovira, Chem. Soc. Rev. 2008, 37, 827-838.

[7] T. Mori, J. Phys. Condens. Matter 2008, 20, 184010.

[8] R. Meerheim, B. Lussem, K. Leo, Proc. IEEE 2009, 97, 1606-1626.

[9] A. P. Kulkarni, C. J. Tonzola, A. Babel, S. A. Jenekhe, Chem. Mater. 2004, 16, 4556-4573.

[10] A. Facchetti, Chem. Mater. 2011, 23, 733-758.

[11] J. D. Servaites, M. A. Ratner, T. J. Marks, Energy Environ. Sci. 2011, 4, 4410.

[12] G. Dennler, M. C. Scharber, C. J. Brabec, Adv. Mater. 2009, 21, 1323-1338.

[13] B. C. Thompson, J. M. J. Fréchet, Angew. Chem. Int. Ed. 2008, 47, 58-77.

[14] B. P. Rand, J. Genoe, P. Heremans, J. Poortmans, Prog. Photovoltaics Res. Appl. 2007, 15, 659-676.

[15] S. Günes, H. Neugebauer, N. S. Sariciftci, Chem. Rev. 2007, 107, 1324-1338.

[16] X. Cheng, M. Caironi, Y.-Y. Noh, C. Newman, J. Wang, M. J. Lee, K. Banger, R. D. Pietro, A. Facchetti, H. Sirringhaus, Org. Electron. 2012, 13, 320-328.

[17] H. Sirringhaus, T. Kawase, R. H. Friend, T. Shimoda, M. Inbasekaran, W. Wu, E. P. Woo, Science 2000, 290, 2123-2126.

[18] Z. Bao, A. Dodabalapur, A. J. Lovinger, Appl. Phys. Lett. 1996, 69, 4108-4110.

[19] B. A. Jones, A. Facchetti, M. R. Wasielewski, T. J. Marks, J. Am. Chem. Soc. 2007, 129, 15259-15278.

[20] H. Bässler, A. Köhler in Unimolecular and Supramolecular Electronics I, Vol. 312 (Ed.: R. M. Metzger), Springer Berlin Heidelberg, 2012, pp. 1-65.

[21] M. Fujitsuka, S. Tojo, M. Shibahara, M. Watanabe, T. Shinmyozu, T. Majima, J. Phys. Chem. A 2011, 115, 741-746. 
[22] A. A. Bakulin, A. Rao, V. G. Pavelyev, P. H. M. van Loosdrecht, M. S. Pshenichnikov, D. Niedzialek, J. Cornil, D. Beljonne, R. H. Friend, Science 2012, 335, 1340-1344.

[23] C. Deibel, T. Strobel, V. Dyakonov, Adv. Mater. 2010, 22, 4097-4111.

[24] X. Guo, N. Zhou, S. J. Lou, J. Smith, D. B. Tice, J. W. Hennek, R. P. Ortiz, J. T. L. Navarrete, S. Li, J. Strzalka, L. X. Chen, R. P. H. Chang, A. Facchetti, T. J. Marks, Nat. Photon. 2013, 7, 825-833.

[25] A. T. Healy, B. W. Boudouris, C. D. Frisbie, M. A. Hillmyer, D. A. Blank, J. Phys. Chem. Lett. 2013, 4, 3445-3449.

[26] M. C. Heiber, A. Dhinojwala, J. Phys. Chem. C 2013, 117, 21627-21634.

[27] H. Tamura, I. Burghardt, J. Am. Chem. Soc. 2013, 135, 16364-16367.

[28] T. A. Madison, G. R. Hutchison, J. Phys. Chem. C 2011, 115, 17558-17563.

[29] A. G. Gagorik, G. R. Hutchison, J. Phys. Chem. C 2012, 116, 21232-21239.

[30] A. G. Gagorik, J. W. Mohin, T. Kowalewski, G. R. Hutchison, J. Phys. Chem. Lett. 2013, 4, 36-42.

[31] T. A. Madison, A. G. Gagorik, G. R. Hutchison, J. Phys. Chem. C 2012, 116, 11852-11858.

[32] M. D. Hanwell, T. A. Madison, G. R. Hutchison, J. Phys. Chem. C 2010, 114, 20417-20423.

[33] C. M. Proctor, M. Kuik, T.-Q. Nguyen, Prog. Polym. Sci. 2013, 38, 1940-1960.

[34] M. Casalegno, G. Raos, R. Po, J. Chem. Phys. 2010, 132, 094705.

[35] F. Wei, L. Liu, L. Liu, G. Li, IEEE J. Photovoltaics 2013, 3, 300-309.

[36] G. Li, L. Liu, F. Wei, S. Xia, X. Qian, IEEE J. Photovoltaics 2012, 2, 320-340.

[37] A. H. Schoen, Infinite periodic minimal surfaces without self-intersections, NASA Technical Report D-5541, 1970. 
The effects of charge delocalization on device efficiency is probed using mesoscale Monte Carlo simulations of charge transport in idealized and isotropic two-phase morphologies. Interfacial charge trapping is drastically reduced when Coulomb interactions are weakened through moderate delocalization (1.0-2.0 nm). Morphological differences become less dominant as charges delocalize.

charge transport

Adam G. Gagorik, Jacob W. Mohin, Tomasz Kowalewski, Geoffrey R. Hutchison*

Effects of Delocalized Charge Carriers in Organic Solar Cells:

Predicting Nanoscale Device Performance from Morphology

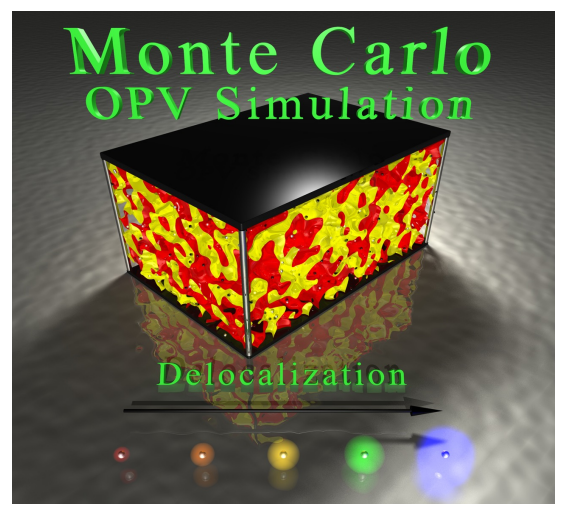


Copyright WILEY-VHC Verlag GmbH \& Co. KGaA, 69469 Weinheim, Germany, 2013.

\section{Supporting Information}

Effects of Delocalized Charge Carriers in Organic Solar Cells: Predicting Nanoscale Device Performance from Morphology

Adam G. Gagorik, Geoffrey R. Hutchison*, Jacob W. Mohin, Tomasz Kowalewski
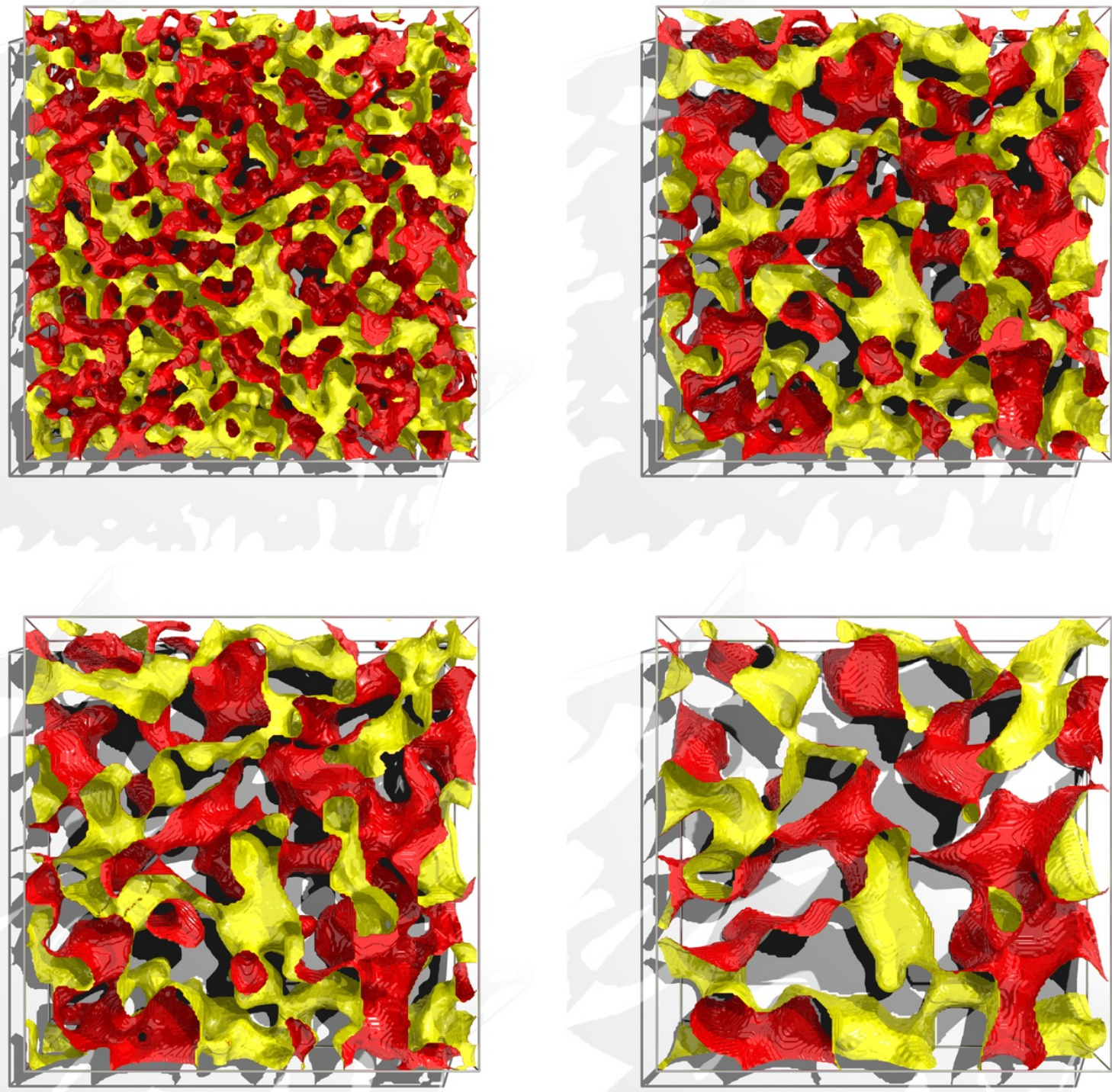

Figure S1: Full isotropic morphologies. 

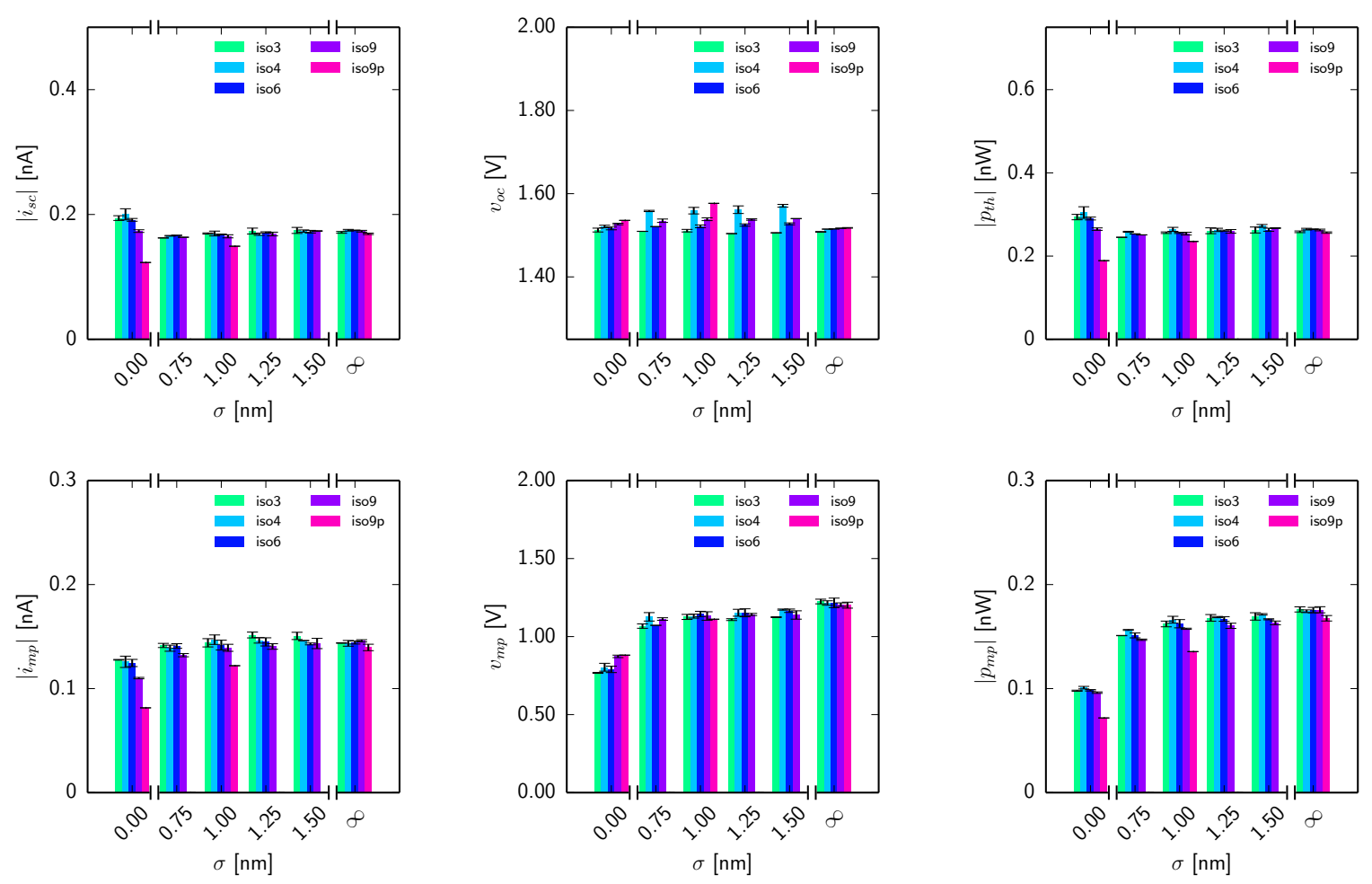

Figure S2: All isotropic IV Curve parameters.

\begin{tabular}{r|rrrrrrrrrr}
$\sigma(\mathrm{nm})$ & $r_{\text {hole }}(\mathrm{nm})$ & $r_{\text {elec } 0}(\mathrm{~nm})$ & $r_{\text {elec } 1}(\mathrm{~nm})$ & $\Delta r(\mathrm{~nm})$ & $E_{0}(\mathrm{eV})$ & $E_{1}(\mathrm{eV})$ & $\Delta E(\mathrm{eV})$ & $\beta \Delta E$ & $P_{a c c}(\%)$ & $H_{a b} P_{a c c}(\%)$ \\
\hline 0.00 & 0 & 1 & 2 & 1 & -0.411 & -0.206 & 0.186 & 7.184 & 0.076 & 0.025 \\
0.25 & 0 & 1 & 2 & 1 & -0.411 & -0.206 & 0.186 & 7.183 & 0.076 & 0.025 \\
0.50 & 0 & 1 & 2 & 1 & -0.393 & -0.206 & 0.167 & 6.460 & 0.156 & 0.052 \\
0.75 & 0 & 1 & 2 & 1 & -0.336 & -0.204 & 0.112 & 4.341 & 1.302 & 0.434 \\
1.00 & 0 & 1 & 2 & 1 & -0.281 & -0.196 & 0.065 & 2.496 & 8.243 & 2.748 \\
1.25 & 0 & 1 & 2 & 1 & -0.237 & -0.183 & 0.034 & 1.313 & 26.913 & 8.971 \\
1.50 & 0 & 1 & 2 & 1 & -0.204 & -0.168 & 0.015 & 0.599 & 54.958 & 18.319 \\
\hline 0.00 & 0 & 1 & 3 & 2 & -0.411 & -0.137 & 0.254 & 9.836 & 0.005 & 0.000 \\
0.25 & 0 & 1 & 3 & 2 & -0.411 & -0.137 & 0.254 & 9.835 & 0.005 & 0.000 \\
0.50 & 0 & 1 & 3 & 2 & -0.393 & -0.137 & 0.236 & 9.112 & 0.011 & 0.000 \\
0.75 & 0 & 1 & 3 & 2 & -0.336 & -0.137 & 0.179 & 6.933 & 0.097 & 0.004 \\
1.00 & 0 & 1 & 3 & 2 & -0.281 & -0.137 & 0.124 & 4.800 & 0.823 & 0.030 \\
1.25 & 0 & 1 & 3 & 2 & -0.237 & -0.135 & 0.082 & 3.180 & 4.159 & 0.154 \\
1.50 & 0 & 1 & 3 & 2 & -0.204 & -0.131 & 0.053 & 2.041 & 12.993 & 0.481
\end{tabular}

Table S1: Energy of moving an electron near a hole. 

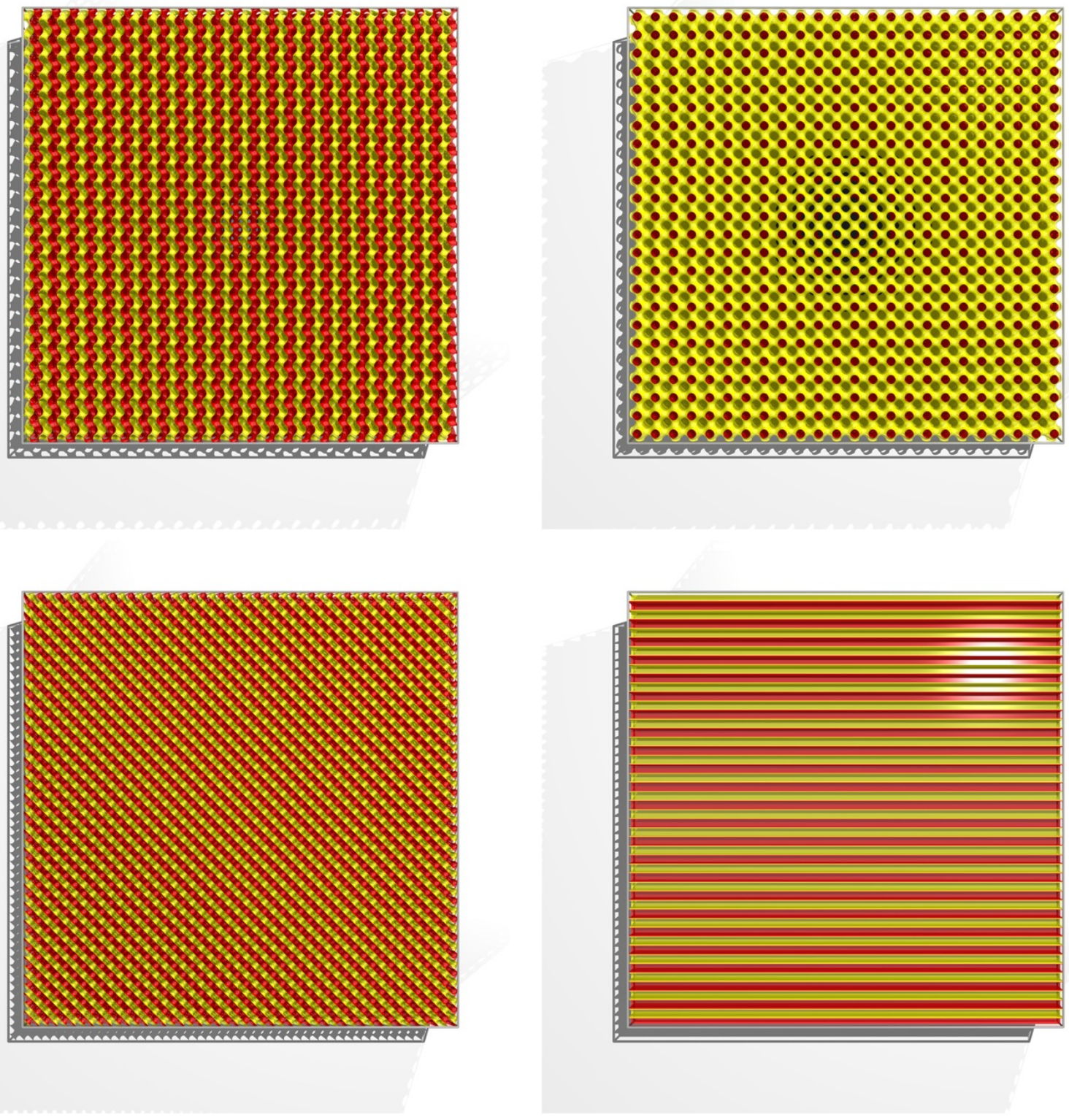

Figure S3: Full ideal morphologies. 

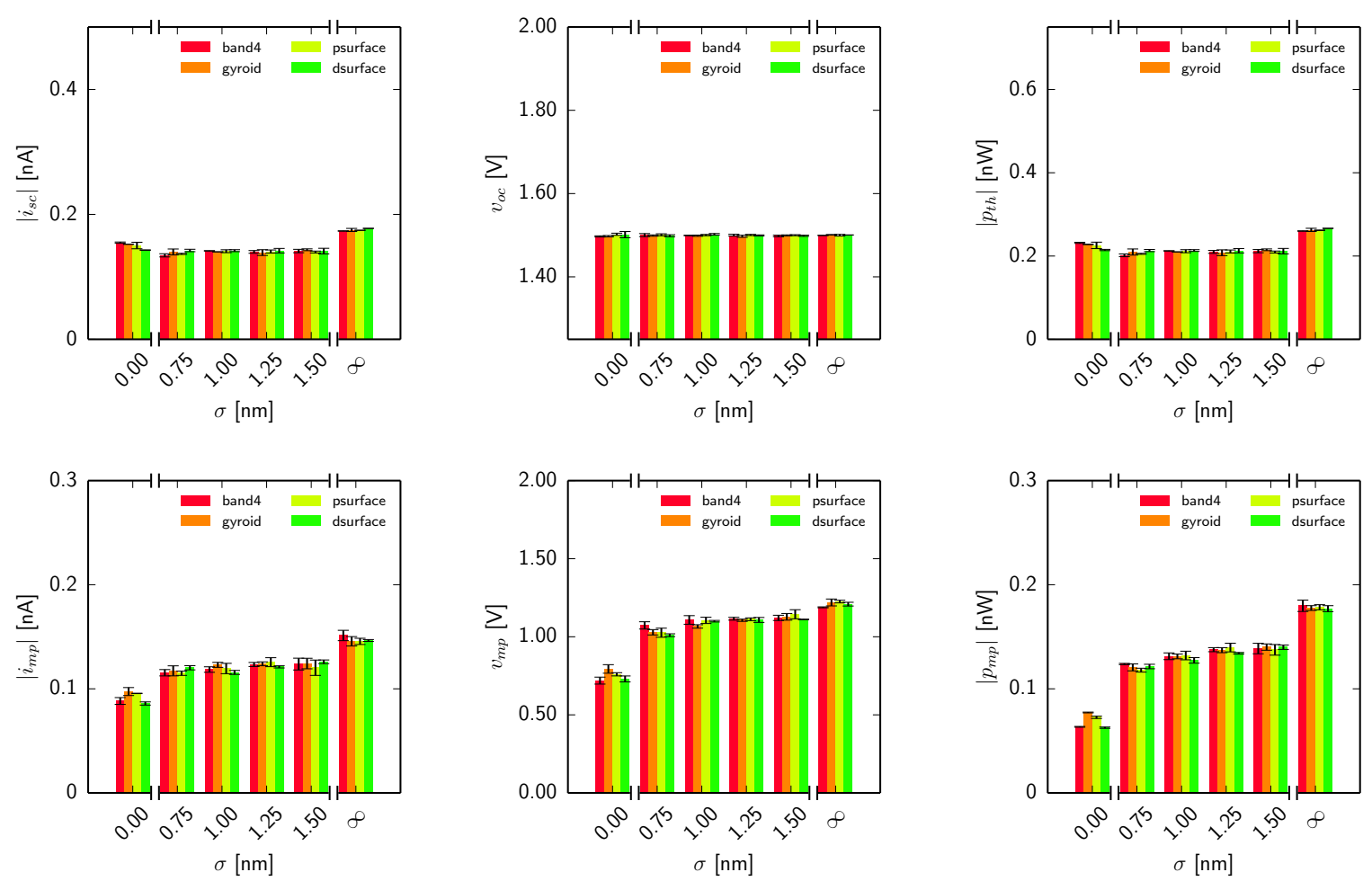

Figure S4: All ideal IV Curve parameters.

No bias

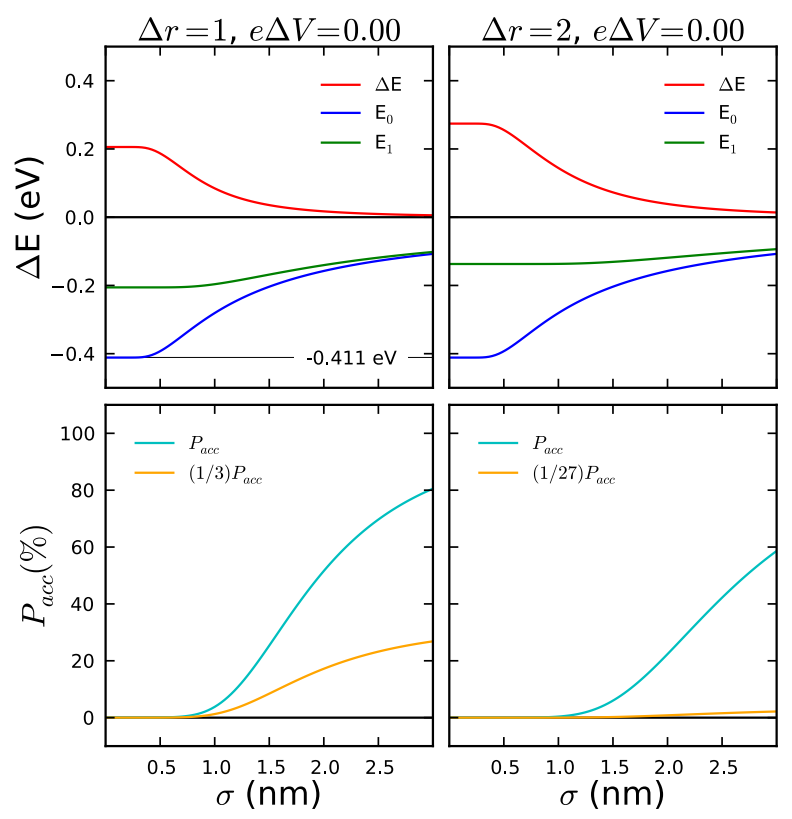

Biased
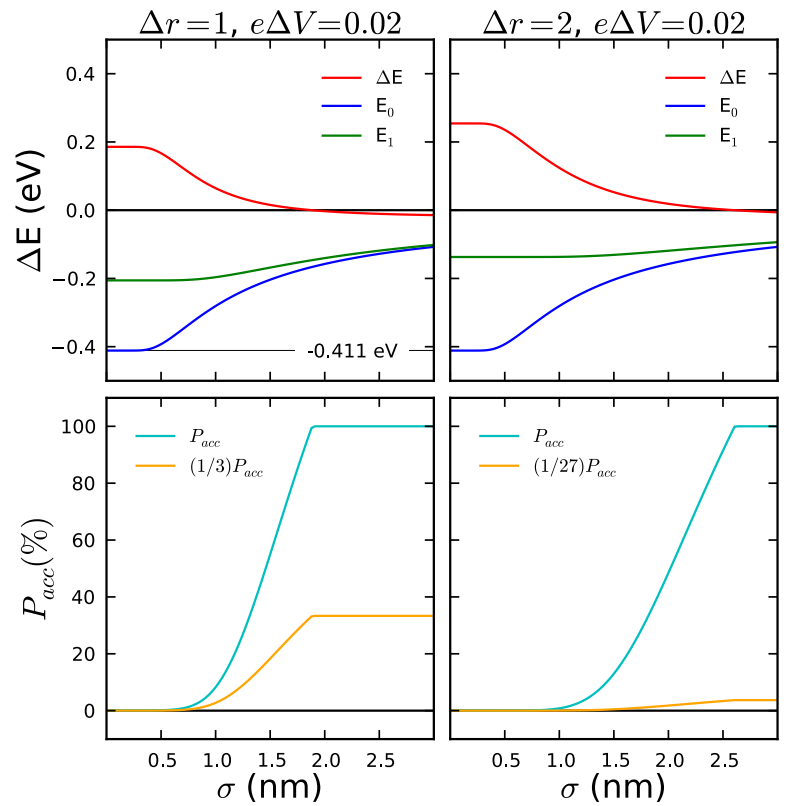

Figure S5: Acceptance probability as a function of sigma. 